\title{
Plan integral de comunicación estratégica para fomentar la participación ciudadana y las prácticas culturales del Cantón Chambo
}

\author{
Cuadrado Samaniego Víctor Hugo \\ victorcuadrado81@gmail.com \\ Licenciado en Comunicación Social con \\ especialidad en desarrollo. \\ Magíster en Gestión Pública.
}

\section{RESUMEN}

Es importante para esta investigación, desarrollar un plan integral de comunicación a través de un producto audiovisual orientado a la población local del Cantón Chambo, capaz de potenciar la participación ciudadana a partir de la intervención local y sus diferentes prácticas culturales. Este plan responde al diseño de una estrategia de comunicación adecuada al contexto digital, la tendencia de consumo de productos y servicios mediáticos, por otra parte son declaradas las investigaciones de tipo exploratoria, descriptiva, explicativa y transeccional, además de métodos científicos, deductivos e inductivos se citan del análisis de contenidos, fuentes estructuradas y no estructuradas de información, para la estrategia metodológica acoge un enfoque cualitativo y cuantitativo desde técnicas como la observación participante, encuestas y entrevistas estructuradas, donde la novedad científica reside en adecuar y contextualizar estrategias comunicacionales a la medida de la población local; de esta manera se concluye que el plan integral basado en un modelo estratégico comunicacional, responde a los intereses del público objetivo, al adoptar, asimilar y proyectar prácticas culturales locales que permitan mayor acceso en calidad y cantidad a la participación ciudadana. Es sugerido socializar la estrategia comunicacional diseñada, atendiendo al propio contexto local y rasgos inherentes el enfoque estratégico de comunicación.

Palabras Claves: Producto audiovisual; estrategia; contexto digital; población local; práctica cultural 


\title{
Comprehensive strategic communication plan to promote citizen participation and cultural practices of the Canton Chambo
}

\begin{abstract}
It is important for this research to develop a comprehensive communication plan through an audiovisual product aimed at the local population of the Canton Chambo, capable of enhancing citizen participation through local intervention and its different cultural practices. This plan responds to the design of a communication strategy appropriate to the digital context, the trend of consumption of media products and services, on the other hand, exploratory, descriptive, explanatory and transectional investigations are declared, as well as scientific, deductive and inductive methods. The content analysis, structured and unstructured sources of information are cited, for the methodological strategy it welcomes a qualitative and quantitative approach from techniques such as participant observation, surveys and structured interviews, where the scientific novelty lies in adapting and contextualizing communication strategies to the measure of local population; In this way, it is concluded that the comprehensive plan based on a strategic communication model responds to the interests of the target audience, by adopting, assimilating and projecting local cultural practices that allow greater access in quality and quantity to citizen participation. It is suggested to socialize the communication strategy designed, taking into account the local context and inherent features of the strategic communication approach.
\end{abstract}

Keywords: Audiovisual product; strategy; digital context; local population; cultural practice.

Artículo recibido: 05 de Abril 2021 Aceptado para publicación: 28 de Mayo 2021 Correspondencia: victorcuadrado81@gmail.com Conflictos de Interés: Ninguna que declarar 


\section{INTRODUCCIÓN}

El rol de la comunicación en el desarrollo de los pueblos, se visibiliza en calidad de herramienta necesaria que surge a partir de una estrategia eficaz que contribuye a lograr objetivos y sostenibilidad de resultados en proyectos. Desde este enfoque, y al referir la comunicación mediática asumida como arista de la comunicación en su sentido amplio, el investigador (Cuadrado, 2007, p.17) expresa que "la promoción de la cultura, de los valores propios, la realización de una síntesis fecunda entre tradición y modernidad, revela otra de las funciones que han sido asignadas para América Latina hacia los medios de comunicación" (p.17).

Más, desde la dimensión sociocultural, la comunicación obliga a generar sus propias estrategias, Tales desafíos son planteados la comunicación social y su abordaje estratégico que como bien argumenta (TRANSMEDIA, 2020, pág. 1), señala:

Debemos intentar comprender los mecanismos socioculturales que entran a dialogar con los avances tecno-científicos y desde ahí poder generar estrategias de comunicación adecuadas a los objetivos que nos guían y siempre respetando las lógicas propias de cada medio y de nuestros receptores, públicos o audiencias. (p.1)

Es decir, la elaboración de estrategias comunicacionales implica un estudio preliminar de las necesidades a rellenar, los objetivos a alcanzar, pues como acertadamente afirma (Pavón, 2019, pág. 2), "si no sabemos cómo queremos alcanzar nuestros objetivos, difícilmente podremos medir el éxito de nuestra estrategia". (p.2). Es preciso reconocer el papel de las herramientas digitales que permiten el flujo constante entre organizaciones y actores la oportuna retroalimentación, sean entre públicos de naturaleza externa o interna.

Entonces, la estrategia comunicacional, está inmersa en el llamado ecosistema digital social, al pretender maximizar sus objetivos y que, en esta investigación, es potenciar la participación ciudadana apelando a las prácticas culturales. Como bien asevera (González, 2020, pág. 2), al referirse:

Y es que el 2020 ha sido un violento punto de inflexión a nivel de comunicación, derivado en buena parte de las medidas de confinamiento dictadas por la pandemia de COVID-19. En un contexto así, no son solo las empresas las que han debido internarse con mayor 
profundidad en las aguas digitales, los clientes potenciales también lo han hecho. La tendencia de una comunicación cada vez más digital se aceleró. En este sentido, uno de los principales retos radica en la transformación digital. Esto implica un sólido entretejido de cuatro componentes: tecnología, procesos, estrategia y personas. (p.2).

Asociado a la comunicación y educación en el mundo digital, la transformación digital implica lógicas participativas entre ciudadanos devenidos usuarios en red, participando en igualdad de condiciones, sean desde sus teléfonos móviles o desde PC. Esta afirmación es compartida con los criterios de (García \& Pérez, 2020, pág. 7), al expresar:

La cuestión que emerge ante un panorama de inexorable penetración tecnológica y conectividad es si se están realizando las acciones y prácticas adecuadas para que esa participación resulte crítica y democrática. [.....]. comunicación y educación se entrelazan para ofrecer aportes significativos en cuatro áreas: la comunicación tratada en el ámbito educativo: el periodismo en el aula y las competencias transmedia; las redes sociales como espacios para la comunicación y la educación: uso de Twitter e Instagram en contexto educativo, bots, desinformación, bulos y competencias digitales; las nuevas generaciones conectadas, y actores emergentes en la comunicación y la educación (p.7)

Referido a estudios sobre la juventud y la cultura digital en la literatura académica, estos caracterizan perfiles de comunidades jóvenes, conectadas y urbanas, que participan en los entornos digitales como lugares de interacción, construcción de la identidad, aprendizaje, ocio, participación política. En el caso específico de Latinoamérica, y asumiendo los preceptos de Ricaurte (2018), manifiesta:

En América Latina es imprescindible entender el sentido de las prácticas digitales juveniles de forma situada y diacrónica, además de promover estudios que expliquen cómo la dimensión socio técnica se encuentra anclada a marcos dominantes que perfilan su apropiación y la producción de subjetividades. (p.1).

Referido al tratamiento de las hipermediaciones en el entorno digital, se precisa por parte del comunicólogo la estrategia a diseñar, al enfatizar entre las diferencias entre el estudio 
de la comunicación digital y la comunicación analógica. Este autor de la presente investigación, se adhiere a los presupuestos esgrimidos por (Ulloa \& Gómez, 2019, pág. 2) donde afirman que:

En este sentido, la teoría de las hipermediaciones abre una nueva área por estudiar que en esta investigación se tuvo como objetivo: construir la teoría de las hipermediaciones y determinar que existen dos tipos de hipermediaciones, que son las sociales y de consumo. (p.2).

\subsection{Relativo al estado del arte en el campo de estudio investigado}

En la presente investigación, se asumen los criterios y presupuestos de (Manfredi, Ufarte, \& Herranz, 2019), referido a la innovación periodística y la sociedad digital; (Martín Barbero, 1984), en cuanto a las contradicciones comunicacionales y sus procesos en América Latina; de (Ordóñez, 2018), asociado a la narrativa y narración en el relato audiovisual en cuando a forma y contenido; (Cruz, Ojalvo, \& Velasteguí, 2019) y el papel de la comunicación en el desarrollo local en especial las estrategias y modelos factibles para la participación y articulación de actores sociales; (Flores, Segura, \& Vanderkast, 2007), relativo a la aproximación a la Sociedad de la Información y del Conocimiento; (Escobar \& Bahamonde, 2020) y (Alba, 2002), referido al cambio cultural y transformación audiovisual asumido desde la visión de la migración de lo tecnológico a lo digital; énfasis en los postulados de (Ricaurte, 2018, pág. 3) y asociado la juventud y cultura digital, en especial cuando expresa que:

En América Latina es imprescindible entender el sentido de las prácticas digitales juveniles de forma situada y diacrónica, además de promover estudios que expliquen cómo la dimensión socio técnica se encuentra anclada a marcos dominantes que perfilan su apropiación y la producción de subjetividades. [....] casos menos visibles de prácticas digitales que exploran la desobediencia epistémica y la resistencia a través de la ocupación tecnológica. (p.3)

Resulta necesario en esta investigación, la presencia de los autores (Hernández, Paz, \& Caballero, 2017, pág. 5), en cuanto a los desafíos de la praxis comunitaria en las políticas públicas de los medios de comunicación latinoamericanos, donde afirman que:

Los medios de comunicación de la región latinoamericana evidencian políticas públicas tergiversadas por las prácticas institucionalizadas. La 
problemática de la participación comunitaria en la producción televisiva subyace en la triada políticas-programas-productos comunicativos. (p.5).

Es imprescindible acotar que, la comunicación desde sus inicios en la humanidad y en calidad de recurso de construcción social, ha sido rol clave al decodificar información generada a través del conocimiento. Desde los constructos simbólicos y el reconocimiento de las prácticas culturales son adoptados los postulados de (Badía et al, 2017, p.8) al expresar:

Periodismo y educomunicación en contextos digitales es un recorrido e incursión profundos -desde la experiencia investigativa de los autorespor un tema que continuará generando expectativas en la misma medida en que los avances cuenticos y tecnológicos contribuyan a la interacción social y al progreso del conocimiento. (p.8).

\subsection{Problematización}

La irrupción de los contextos digitales en zonas de bajo-medio desarrollo en la adopción de las Tics, aparejado al confinamiento de la pandemia, contrae un reto para el desarrollo y fortalecimiento cultural, así como la responsabilidad del estado en prevalecer los derechos ciudadanos y la práctica de una política pública cultural a nivel de los municipios. Se evidencia del incremento del uso de herramientas de contexto digital por las nuevas generaciones, como medio de comunicación y socialización para su desarrollo. Esta realidad, implica la adopción de estrategias comunicacionales, hoy inexistentes en el campo de estudio, Cantón Chambo, provincia Chimborazo.

Así, se precisa determinar el tipo de estrategia comunicacional que permita la participación e involucramiento de la población local, implícita en todas sus generaciones, para el desarrollo y fortalecimiento de sus prácticas culturales; de la ausencia de análisis desde la población local determinar cuáles medios de comunicación con tendencia a contexto digital, están mediando para el desarrollo de la participación ciudadana en las prácticas culturales; la ausencia de la evidencia del grado de planificación y los diferentes tipos de actividades que realiza el GAD Municipal del Cantón Chambo para el desarrollo e involucramiento de la participación ciudadana en el fortalecimiento de sus prácticas culturales. La problematización es descrita en el árbol de problemas de la figura 1. 


\section{Figura 1. Árbol de problemas}

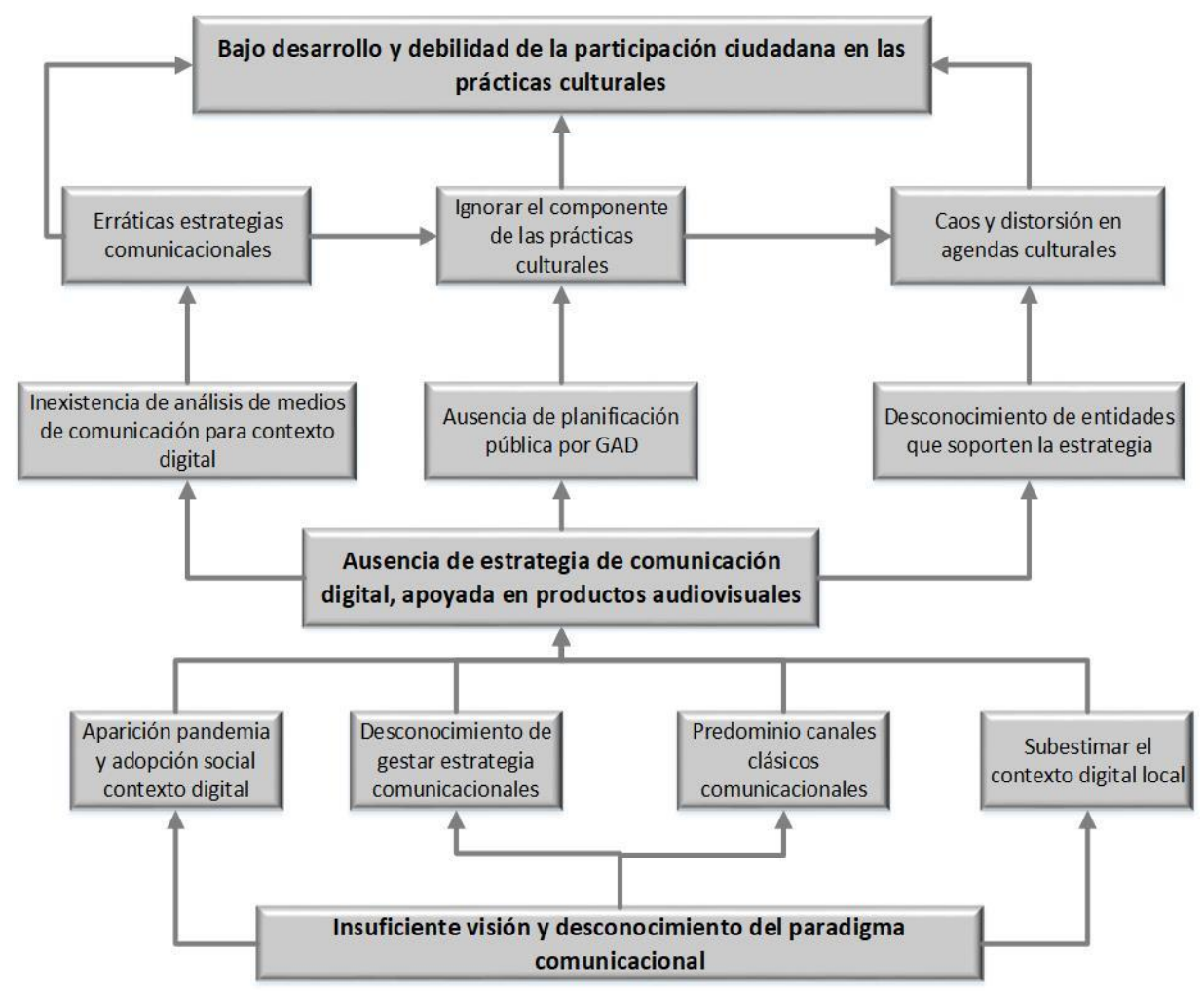

Fuente: Víctor Cuadrado, 2021.

Entonces, al revelar el problema científico, se declara siguiente pregunta: ¿Es posible diseñar estrategia de comunicación digital apoyada en productos audiovisuales a implementar para el desarrollo y fortalecimiento de la participación ciudadana en las prácticas culturales del Cantón Chambo?

De tal manera, es preciso descifrar diferentes tipos de estrategias que permitan determinar la incidencia de la política pública cultural, y a la vez reconocer los diferentes espacios y escenarios donde la ciudadanía se desenvuelve dentro de un contexto social, desarrollado en las diferentes agendas culturales que se emiten a través del GAD Municipal del Cantón Chambo.

De las preguntas específicas que soportan esta investigación, se cita el cómo se construye el proceso de participación ciudadana para el fortalecimiento de las prácticas culturales en el Cantón Chambo; de la existencia medios de comunicación tradicionales y de contexto digital que permitan el desarrollo y difusión de las prácticas culturales; de la existencia de entidades adscritas al GAD Municipal del Cantón Chambo que permitan el desarrollo y fortalecimiento de la participación ciudadana y de la evidencia en cuanto al 
plan o agenda de desarrollo Cultural en el GAD Municipal del Cantón Chambo, que permita manejar y solventar los recursos para la consecución de las prácticas culturales. Se adicionan a estas preguntas específicas, de la factibilidad de diseñar estrategia comunicacional que responda las variables de las prácticas culturales y la participación ciudadana; de la vialidad para recrear procedimiento de la estrategia en cuestión; de la capacidad de generalizar el modelo estratégico comunicacional, atendiendo a las particularidades y contextos locales a través de canales académicos y públicos.

Se parte de la hipótesis que, al diseñar estrategia de comunicación digital apoyada en productos audiovisuales a implementar para el desarrollo y fortalecimiento de la participación ciudadana en las prácticas culturales del Cantón Chambo, se logra la estrategia comunicacional, el empoderamiento como sujeto y objeto de los cambios sociales exigidos, todo ello desde el contexto digital.

\subsection{Objetivos y justificación de la investigación}

El diseño de la estrategia de comunicación digital apoyada en productos audiovisuales a implementar para el desarrollo y fortalecimiento de la participación ciudadana en las prácticas culturales del Cantón Chambo, constituye el objetivo principal de esta investigación.

Los objetivos específicos están centrados en determinar cómo se construye el proceso de participación ciudadana para el fortalecimiento de las prácticas culturales en el Cantón Chambo; de evidenciar y potenciar medios de comunicación tradicionales y de contexto digital que permitan el desarrollo y difusión de las prácticas culturales; del apoyo y desarrollo de entidades adscritas al GAD Municipal del Cantón Chambo para el fortalecimiento de la participación ciudadana y de la evidencia en cuanto al plan o agenda de desarrollo Cultural en el GAD Municipal del Cantón Chambo, de tal manera manejar y solventar los recursos para la consecución de las prácticas culturales.

Se complementan estos objetivos específicos con la factibilidad de diseñar estrategia comunicacional que responda las variables de las prácticas culturales y la participación ciudadana; de la vialidad para recrear procedimiento de la estrategia en cuestión; de la capacidad de generalizar el modelo estratégico comunicacional, atendiendo a las particularidades y contextos locales a través de canales académicos y públicos. Se parte de la hipótesis que, la propuesta del modelo de estrategia comunicacional, aporta a la 
participación ciudadana y empoderamiento como sujeto y objeto de los cambios sociales exigidos, desde el contexto digital.

La justificación de la investigación, es asumida desde los presupuestos formulados por (Hernández, Fernández, \& Baptista, 2006, pág. 82) en la etapa del diseño de investigación y su conveniencia, pues la necesidad de revitalizar estrategias comunicacionales, desde el contexto digital en los momentos actuales, aporta un alto sentido de pertinencia social. Tal afirmación, a partir del giro social por la pandemia, y las nuevas tendencias en el uso de las Tics, obliga a reformular herramientas comunicacionales basadas en estrategias bien formuladas. Así, la justificación socioeconómica se expresa al aportar una herramienta que eleva la calidad e interacción comunicacional social, permite abaratar gastos en cuanto a la oportunidad de los mensajes y foros de discusión, imprime sesgo cultural al asumir las prácticas locales, permite como canal participativo social.

Referido a la relevancia social de la investigación, estriba en la mayor participación ciudadana local en el contexto digital al adoptar un producto audiovisual a la medida de la estrategia comunicacional diseñada. Los actores beneficiados son las personas, la radio, el entorno, la sociedad en general.

Las implicaciones prácticas de esta investigación residen en la solución de perfeccionar la comunicación audiovisual en el contexto digital, atendiendo a la elevación de la participación ciudadana desde los presupuestos de la práctica cultural bidireccional. La novedad científica reside en la propia estrategia al contener un producto audiovisual que responde, desde el contexto digital a la estrategia comunicacional local. Así, el árbol de objetivos se refleja en la figura 2. 
Figura 2. Árbol de objetivos

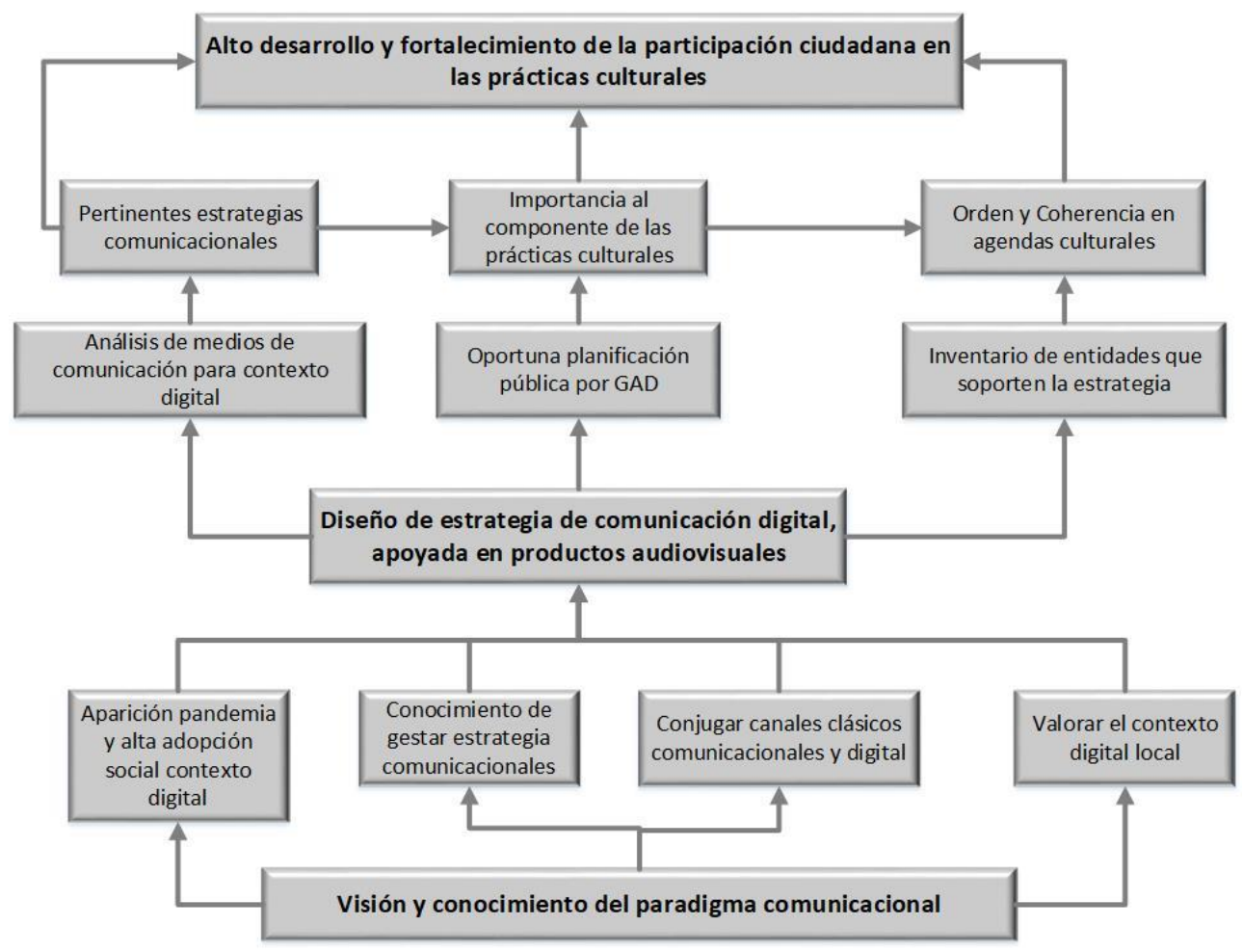

Fuente: Víctor Cuadrado, 2021.

El aporte comunicacional será el resultado de un ejercicio dialógico, serio y a la vez ejecutable para fomentar actividades que conllevan a conseguir objetivos propuestos para la posteridad con el fin de proyectar desde un medio digital "Plus Tv Comunicación Digital" y con la colaboración con el GAD Municipal del Cantón Chambo y sus entidades adscritas datos específicos de proyectos y prácticas culturales que permitan el desarrollo y fortalecimiento de la participación ciudadana en el sector.

\section{METODOLOGÍA DE LA INVESTIGACIÓN}

Esta investigación, declara su carácter científico, pues se define como la serie de pasos que conducen a la búsqueda de conocimientos mediante la aplicación de métodos y técnicas de corte científico.

\subsection{Tipos de estudios adoptados}

Se adoptan y declaran estudios de tipo exploratorio, pues el campo de la estrategia comunicacional en el contexto local es poco estudiado y no ha sido abordado con la profundidad requerida con anterioridad. Se evidencian insuficientes fuentes referenciales en el Ecuador sobre este campo investigativo, enfatizando la ausencia de estudios y modelos comunicacionales estratégicos locales con la praxis cultural y orientado a la 
participación ciudadana. Así, esta investigación aporta contenido pertinente el problema declarado, y aún poco estudiado en el contexto local, a partir de la propuesta del modelo estratégico comunicacional gestado.

La investigación descriptiva permite identificar conceptos y variables que permiten establecer prioridades para investigaciones posteriores o sugerir afirmaciones en cuanto a la adecuación de instrumentos para la determinación de estrategias comunicacionales, caracterizadas por su intención y variables contenidas. La gestión informacional ejecutada, revela que existen nichos no investigados acerca de la disponibilidad de estudios de esta naturaleza, donde adoptar las mejores prácticas y adecuar en diversos contextos locales y territoriales.

Énfasis en esta investigación en el marco metodológico, que a tenor de (Hurtado \& Toro, 2007, pág. 90), al afirmar que "el diseño del marco metodológico constituye la medula de la investigación, se refiere al desarrollo propiamente del trabajo investigativo" (p.90). El estudio de tipo descriptivo, se aplica a tenor de los postulados de (Hernández, Fernández, \& Baptista, 2006, pág. 119) donde "la investigación descriptiva busca especificar propiedades, características y rasgos importantes de cualquier fenómeno que se analice" (p.119).

Así, se especifica el modelo de la estrategia comunicacional, revelado en fases y acciones específicas según algoritmo. Ello permite estructurar las fases y pasos de ejecución, además de la mejora para posteriores investigaciones análogas, y de tal manera, es posible evaluar dimensiones y componentes del problema científico. Referido al estudio explicativo, se logra desbordar la mera descripción de conceptos y fenómenos asociados a la estrategia comunicacional, estableciendo relaciones entre estos a partir del instrumento comunicacional implícito en la estrategia. De tal manera, pueden responderse o explicarse enfoques y visiones desde disímiles campos de estudio, como es el comunicacional, social, político, local, intercultural.

Referido a la factibilidad de realización de esta investigación, y asociado al proyecto factible a tenor de (Arias, 2006, pág. 134), el cual señala que "se trata de una propuesta de acción para resolver un problema práctico o satisfacer una necesidad. Es indispensable que dicha propuesta se acompañe de una investigación, que demuestre su factibilidad o posibilidad de realización" (p.134). Precisamente, la investigación parte de un estudio de caso que satisface la posibilidad de realización. 
Referido a la investigación estudio de caso o de campo, es declarado y ejecutado a tenor de criterios de (Roldán, 2000), en calidad de análisis sistemático de problemas con el propósito de detallarlos, explicar sus causas y efectos, entender su naturaleza y los factores contribuyentes y predecir su ocurrencia en el propio medio donde se suscitan los eventos investigados.

Así, este estudio parte del análisis de variables donde contexto digital, estrategia de comunicación, prácticas socioculturales, participación ciudadana, se enlazan sistémicamente a la hora de crear el producto audiovisual como expresión de la estrategia comunicacional.

\subsection{Métodos científicos declarados en la investigación}

Asociado a los métodos científicos declarados, se mencionan el método de análisis y síntesis bibliográfico de las fuentes estructuradas y no estructuradas, logrando conocer y contextualizar las mejores prácticas y estado del arte del campo de estudio y en específico del problema científico; método de consulta a expertos, al apelar a especialistas que evacuen las dudas y permitan reorientar el experimento, que provean del criterio de validez de la propuesta del conocimiento; método de las contradicciones dialécticas, lo que permite descubrir la génesis del problema científico y los elementos conflictuales que en ellos se revelan, desmembrando los efectos y razones.

Se adicionan el método del enfoque sistémico, permitiendo enlazar, relacionar de manera inter e intra disciplinar los procesos implícitos en este modelo, adoptando las relaciones y prácticas dependientes entre estos.

\subsection{De la operacionalización de las variables}

Se adopta como variable independiente la estrategia comunicacional desde los presupuestos de la práctica cultural, y la variable dependiente el grado de desarrollo y fortalecimiento de la participación ciudadana en las prácticas culturales.

\section{DESARROLLO}

Crear producto comunicacional en esta investigación, significa responder a los objetivos planteados; incluir las variables de corte social y comunicacional, así como las dimensiones que comprenden la estrategia; ejecutar un diagnóstico desde el enfoque de gestión. La figura 3, permite visualizar el enfoque de este modelo. 
Este modelo implica declarar las variables o dimensiones adoptadas en la estrategia comunicacional. Las variables han de explicitarse en el algoritmo descriptivo que soporta el modelo en cuestión. Entones, la meta es gestar estrategia comunicacional en el contexto virtual, orientada a las prácticas culturales, promoviendo la participación ciudadana.

En cuando a la Fase 1, resulta el fundamento de la planeación estratégica. En esta fase se aplica FODA estratégico y así reconocer las oportunidades fortalezas existentes en el entorno local, así como las amenazas y fortalezas que conspiran contra el éxito del proyecto estratégico comunicacional. Sólo entonces, es posible gestar el conjunto de estrategias y acciones, capaces de aunar voluntades, minimizar riesgos y garantizar un producto audio visual con los requerimientos exigidos.

Es imposible determinar objetivos y acciones, -afirma el autor de esta investigación-, sin el previo estudio del entorno, enunciar políticas y objetivos estratégicos. Significa que, se minimiza el empirismo en la construcción de la estrategia comunicacional a través de un modelo de gestión comunicacional estructurado, coherentes, sistémico y participativo.

Figura 3. Modelo de la estrategia comunicacional

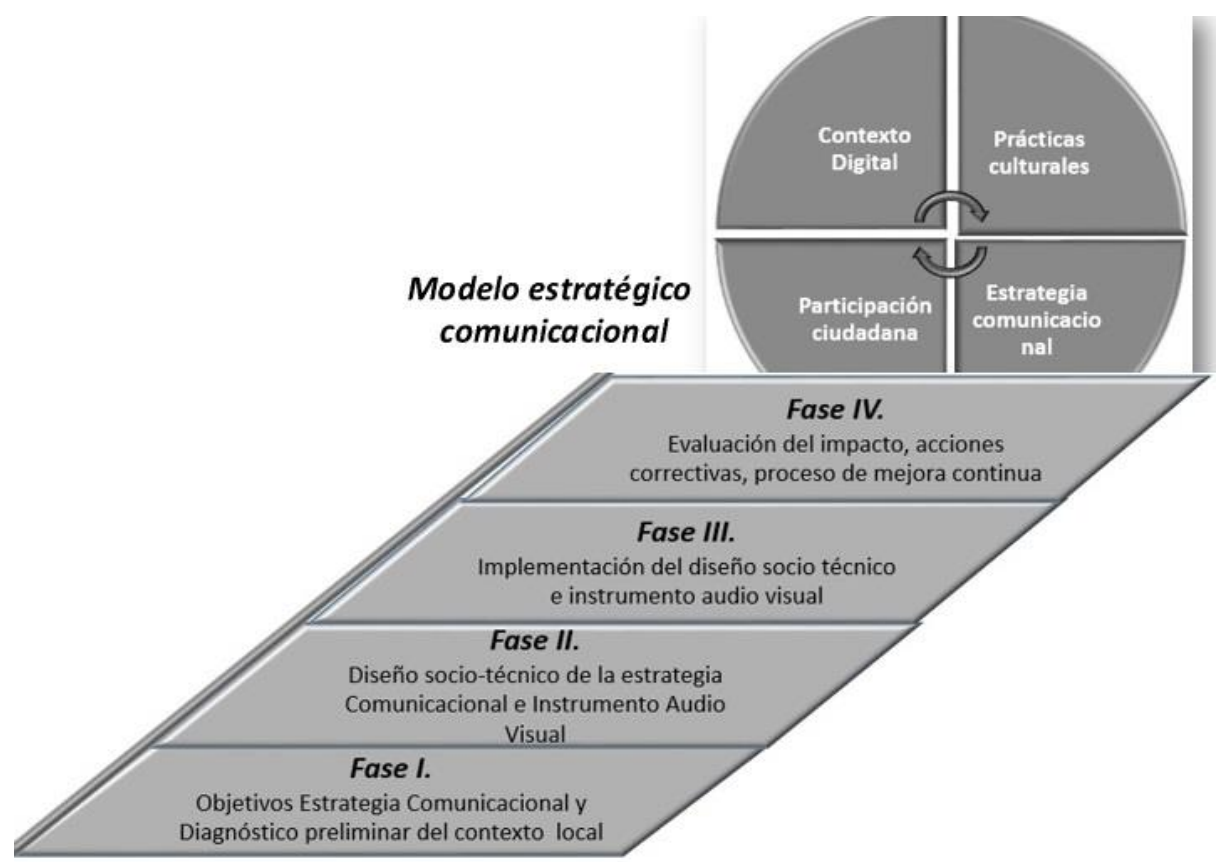

Fuente: Víctor Cuadrado, 2021.

La Fase II del diseño, desde la visión socio-técnica, comprende las variables, recursos, componentes social y cultural, interrelaciones sociales y otros factores orientados a las prácticas culturales. Este término acuñado de socio-técnico, por cuanto la estrategia 
comunicacional se elabora desde las necesidades y presupuestos sociales comunicacionales, y lo técnico reside en los instrumentos, canales y medios para implementar esta esta estrategia. Énfasis en el diseño del producto audio-visual, a la medida de los requerimientos y posibilidades locales.

Referido a la Fase III, la implementación, es preciso monitorear el grado de ejecución de cada acción implícita en el diseño, puesto que la realidad implica la reorientación de ideas, innovación en cuanto a soluciones técnicas y operativas. Ha de ajustarse el instrumento de manera tal, que satisfaga expectativas, tanto del público virtual conectado, como a los presupuestos del diseño.

No es viable diseñar, implementar y declarar exitosa la estrategia comunicacional, a través del producto audiovisual, de no evaluarse el impacto, corregir no conformidades y aplicar acciones correctivas. Así, en la Fase IV, se incorpora al modelo el principio de la mejora continua establecida en los modelos de gestión y aseguramiento de la calidad.

Del modelo descrito, se precisa estructurar cada dimensión y acciones, al velar por la organicidad y la coherencia desde el diagnóstico hasta la evaluación final. Entonces, se impone crear algoritmo que ilustre "el cómo" llevar a cabo el modelo estratégico comunicacional. La figura 4, refleja este algoritmo, cuyo flujo estructurado en cuatro etapas o dimensiones, guían al investigador en la construcción del modelo estratégico comunicacional.

Figura 4. Algoritmo del modelo estratégico comunicacional

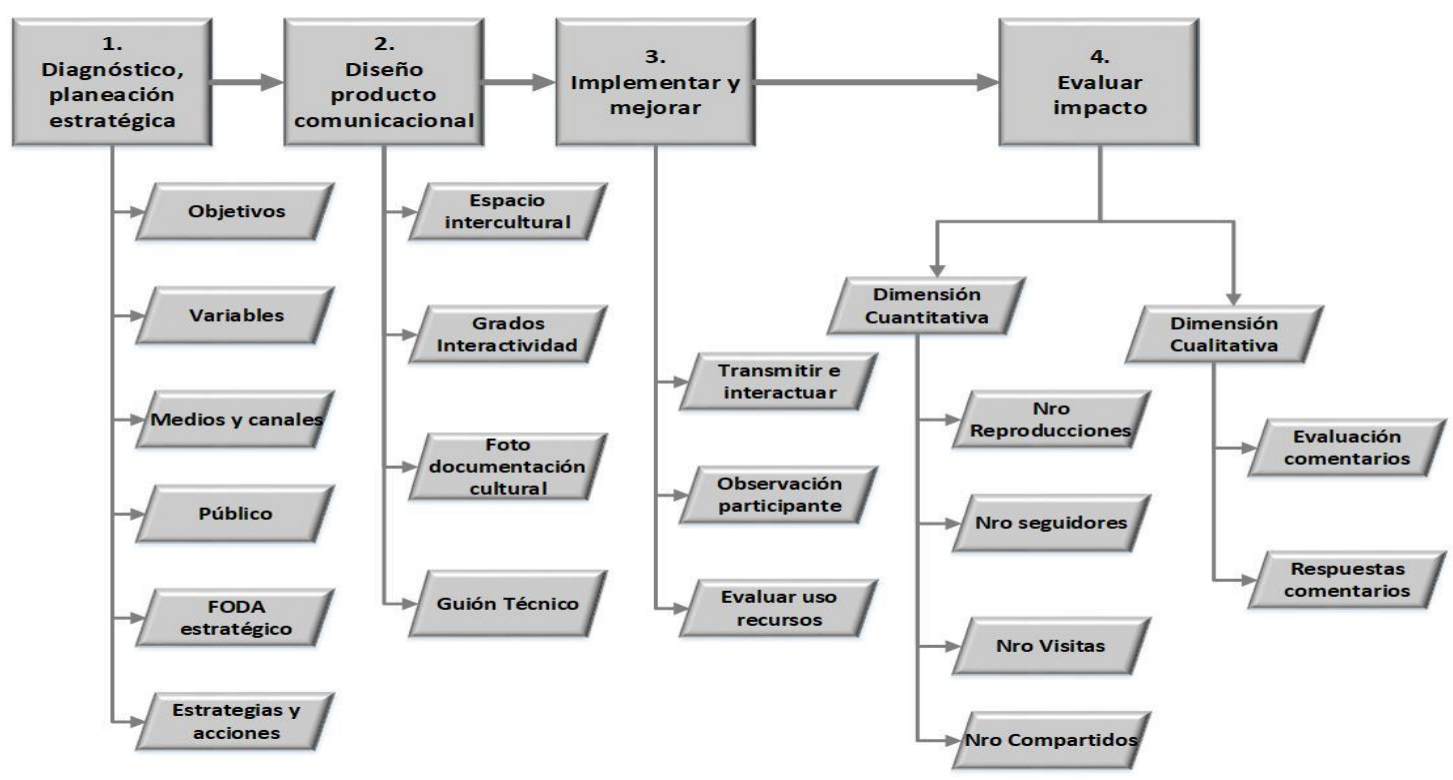

Fuente: Víctor Cuadrado, 2021 
El término "estratégico", comienza desde la planeación, donde los propósitos y objetivos a alcanzar han de estar perfectamente enunciados. Sobre este fundamento, se adoptan las variables de corte social, cultural, comunicacional, históricas, y otras que componen la estrategia comunicacional. Del hardware existente en la localidad, de inventaría y cualifica en cuanto a canales y medios. Otro aspecto es la definición de los públicos metas y abordajes comunicacionales.

Se impone entonces, y con el enfoque participativo de los actores involucrados en este proyecto, crear la FODA que tribute a una matriz estratégica. De esta matriz estratégica, son gestadas, consensuadas y aprobadas las acciones que minimicen el riesgo del diseño posterior, y aproveche las oportunidades del entorno. El diseño del producto comunicacional comprende el campo técnico, donde análisis del espacio intercultural, la adopción de los grados de interactividad con el público, aplicar a la observación participante en calidad de identificación y fusión con las prácticas culturales, permiten la participación ciudadana efectiva.

Entonces, elaboración de guiones técnicos y el soporte documental y fotográfico, enriquecen el conocimiento, revalorización y rescate de las prácticas culturales, incitando a la audiencia a participar en calidad de objeto/sujeto en la preservación, enraizamiento y mantenimiento de estas prácticas. No obstante, a la hora de implementar el producto audiovisual, donde se transmite e interactúa con los públicos en el contexto virtual, ha de evaluarse el uso de los recursos, localizar donde y cuando mejorar, evidenciar no conformidades respecto al diseño, o al criterio del público, y adoptar acciones de mejora. La evaluación del proyecto estratégico comunicacional, implica el análisis de lo cualitativo y cuantitativo. Este monitoreo perenne, exige del reconocimiento y aceptación de la realidad expresada por los públicos que interactúan. Es la evaluación, un ejercicio de retroalimentación que perfecciona, reorienta y redirecciona el diseño del producto comunicacional.

\section{CONCLUSIONES}

Sobre la base de los objetivos propuestos en esta investigación, se concluye que ha sido diseñada la estrategia de comunicación digital, apoyada en productos audiovisuales a implementar para el desarrollo y fortalecimiento de la participación ciudadana en las prácticas culturales del Cantón Chambo, constituye el objetivo principal de esta investigación. 
Se ha determinado cómo se construye el proceso de participación ciudadana para el fortalecimiento de las prácticas culturales en el Cantón Chambo además de evidenciar y potenciar medios de comunicación tradicionales y de contexto digital que permitan el desarrollo y difusión de las prácticas culturales.

Se ha logrado determinar del apoyo y desarrollo de entidades adscritas al GAD Municipal del Cantón Chambo para el fortalecimiento de la participación ciudadana y de la evidencia en cuanto al plan o agenda de desarrollo Cultural en el GAD Municipal del Cantón Chambo, de tal manera manejar y solventar los recursos para la consecución de las prácticas culturales.

Durante el proceso de aplicabilidad del instrumento, se demuestra la factibilidad de diseñar estrategia comunicacional que responda las variables de las prácticas culturales y la participación ciudadana, así como la vialidad para recrear procedimiento de la estrategia en cuestión. Se demuestra de la capacidad de generalizar el modelo estratégico comunicacional, atendiendo a las particularidades y contextos locales a través de canales académicos y públicos.

\section{REFERENCIAS BIBLIOGRÁFICAS}

Alba, G. (2002, septiembre 15). Cambio cultural y transformación audiovisual. De la mediación tecnológica a la migración digital. Revista Signo y Pensamiento. Vol. 21. Nro 41. Pontificia Universidad Javeriana. Recuperado de: https://revistas.javeriana.edu.co/index.php/signoypensamiento/article/view/5203

Arias F. G. (2006). El proyecto de la Investigación. Introducción a la metodología científica. Caracas. Editorial Episteme. Recuperado de: https://universoabierto.org/2017/05/22/el-proyecto-de-investigacionintroduccion-a-la-metodologia-cientifica/

Badía V., A., Gómez M.; M.; Molina C., 1.; Gárciga R., M.; Villota O., W.; Lambert S.; Zambrano C., M. (2017). Periodismo y educomunicación en contextos digitales. Universidad Católica de Santiago de Guayaquil. Guayas, Ecuador. ISBN 9789942-769-23-7 Recuperado de: https://editorial.ucsg.edu.ec/editorial/cienciassociales-y-humanidades/96-periodismo-y-educomunicacon-en-contextosdigitales.html 
Cruz Santa P., D.; Ojalvo M., V.; Velasteguí L., E. (2019). Papel de la comunicación en el desarrollo local. Estrategias y modelos factibles para la participación y la articulación de actores sociales. Revista Electrónica Ciencia Digital, 3(2), 336352. doi:https://doi.org/10.33262/cienciadigital.v9i2.401. recuperado de: http://cienciadigital.org/revistacienciadigital2/index.php/CienciaDigital/article/vi ew/401

Cuadrado S., V.H. (2007). Ensayo de Investigación para obtención de título de Licenciatura en Comunicación Social. La Comunicación como Estrategia de desarrollo en las prácticas culturales del Cantón Chambo.

Escobar, A.; Bahamonde, L. (2020). Innovación en emprendimientos periodísticos y de comunicación frente a las nuevas tendencias digitales. URU, Revista de Comunicación y Cultura(3), 83-100. doi:https://doi.org/10.32719/26312514.2020.3.6. Recuperado de: https://revistas.uasb.edu.ec/index.php/uru/article/view/1491.

Flores P., A. L.; Segura G., G.; Venderkast, E. (2007). Una aproximación a la sociedad de la Información y del Conocimiento. SEMANTIC SCHOLAR. Corpus ID: 161445013. Recuperado de: https://www.semanticscholar.org/paper/Unaaproximaci\%C3\%B3n-a-la-Sociedad-de-la-Informaci\%C3\%B3n-y-PachecoSegura/e0b9e87887ba3e7209e23747d8994605c8fa85e3

García R. R.; Pérez E., A. (2020). Comunicación y Educación en un mundo digital y conectado. Presentación. Icono 14, 18(2), 1-15. doi: https://doi.org/10.7195/ri14.v18i2.1580. Recuperado de: https://icono14.net/ojs/index.php/icono14/article/view/1580

González, I. (actualizado julio, 2020). Qué es la comunicación digital y por qué es importante en las empresas. Audiencias Digitales. Recuperado de: https://ilifebelt.com/que-es-comunicacion-digital-y-por-que-es-importante-enlas-empresas/2016/09/

Hernández A. E.; Paz E, L.; Caballero R., M. (2017). Desafíos de la praxis comunitaria en las politicas públicas de los medios de comunicacion latinoamericanos. Comunicación Revista Internacional de Comunicación Audiovisual, Publicidad y Estudios Culturales, (15), 17-25. Recuperado de: http://www.revistacomunicacion.org/pdf/n15/Articulos/A2_Hernandez-Paz- 
Caballero-Desafios-de-la-praxis-comunitaria-en-las-politicas-publicas-de-losmedios-de-comunicacion-latinoamericanos.pdf

Hernández S.; Fernández, A.; y Baptista N. (2006): Metodología de la Investigación. Quinta Edición. Mc GRAW-HILL/INTERAMERICANA DE EDITORES. S.A. DE C.V. Recuperado de: https://www.esup.edu.pe/descargas/dep_investigacion/Metodologia\%20de\%20la \%20investigaci\%C3\%B3n\%205ta\%20Edici\%C3\%B3n.pdf

Hurtado, I. Toro, G. (2007). Paradigmas y métodos de investigación en tiempos de cambio. Episteme. (5ta Edición) Valencia. Recuperado de: https://es.scribd.com/document/371098034/Hurtado-y-Toro-paradigmas-yMetodos-de-Investigacion-en-Tiempos-de-Cambio

Manfredi S., J. L.; Uferte R., M. J.; Herranz, J. M. (2019). Innovación periodística y sociedad digital: Una adaptación de lso estudios de periodismo. Revista Latina. DOI. : 10.4185/RLCS-2019-1402. Recuperado de: http://www.revistalatinacs.org/074paper/1402/85es.html

Martín B., J. (1984). De la Comunicación a la Cultura: perder el "objeto" para ganar el proceso. Revista Signo y Pensamiento, Vol.31, Nro. 60. Recuperado de: http://www.scielo.org.co/scielo.php?script=sci_abstract\&pid=S0120$48232012000100006 \& \operatorname{lng}=\mathrm{en} \& \mathrm{nrm}=\mathrm{iso} \& \ln \mathrm{ln}=\mathrm{es}$

Ordóñez, G. (2018). Narrativa y narración en el relato audiovisual: Apuntes para la distinción de forma y contenido. UASB. Ecuador. Recuperado de: http://repositorio.uasb.edu.ec/handle/10644/6404

Pavón, M (Octubre, 2019). Una estrategia de comunicación digital enfocada en resultados. Blog. Recuperado de: https://blog.ida.cl/estrategia-digital/estrategiacomunicacion-digital-enfocada-en-resultados/

Ricaurte Q., P. (2018). Jóvenes y cultura digital: abordajes críticos desde América Latina. Chasqui. Revista Latinoamericana de Comunicación, 13-28. doi: https://doi.org/10.16921/chasqui.v0i137.3664. Recuperado en : https://revistachasqui.org/index.php/chasqui/article/view/3664

Roldan García, J. L. (2000). Cómo elaborar un proyecto de investigación. Universidad de Valladolid. España. Recuperado de: 
https://alojamientos.uva.es/guia_docente/uploads/2013/429/52504/1/Documento. pdf

TRANSMEDIA (abril, 2020). Una introducción a las estrategias de comunicación digital. Tecnicatura Superior Comunicación Social. Buenos Aires. Argentina. Recuperado de: https://perio.unlp.edu.ar/catedras/transmedia1/2020/04/23/unaintroduccion-a-las-estrategias-de-comunicacion-digital/

Ulloa L. L. Gómez M., M. (2019). Hipermediaciones que rigen en la comunicación de jóvenes universitarios de Ecuador en Facebook. Revista Ibérica de Sistemas y Tecnologías de la Información, 152-164. Obtenido de https://www.researchgate.net/publication/336070481 